\title{
Habilidades gerenciales como estrategia de competitividad empresarial en las pequeñas y medianas empresas (Pymes)
}

\author{
ALMA BRENDA LEYVA CARRERAS ${ }^{a}$, \\ JOEL ENRIQUE ESPEJEL BLANCO ${ }^{b}$, \\ JUDITH CAVAZOS ARROYOC
}

\begin{abstract}
RESUMEN Esta investigación tiene como objetivo medir la influencia de las habilidades gerenciales como factor interno en la competitividad empresarial que perciben los empresarios de las pequeñas y medianas empresas (Pymes) de Hermosillo, Sonora, México. Para tal efecto, se utilizó una metodología mixta con el fin de poder recolectar, analizar y vincular los datos cuantitativos y cualitativos, y responder al planteamiento de la investigación con mayor evidencia científica. La primera fase fue exploratoria mediante entrevista a panel de expertos usando el método Delphi, para contextualizar la problemática objeto de análisis y validar el instrumento de medida. En la segunda fase se muestrearon 108 Pymes y se realizó un análisis estadístico utilizando modelos de ecuaciones estructurales (PLS) para obtener resultados. Las conclusiones demostraron que las habilidades gerenciales son un factor interno de las Pymes que influyen considerablemente en su competitividad empresarial.
\end{abstract}

PALABRAS CLAVE competitividad empresarial, gerente, habilidades gerenciales, pequeñas y medianas empresas.

\section{HISTORIA DEL ARTÍCULO}

¿CÓMO CITAR?:

Leyva-Carreras, A.B., EspejelBlanco, J.E. \& Cavazos-Arroyo, J. (2017). Habilidades gerencial es como estrategia de competitividad empresarial en las pequeñas y medianas empresas (Pymes). -Perspectiva Empresarial, 4(1), 7-22. http://dx.doi.org/10.16967/rpe. v4n1a1

RECIBIDO: 20 de mayo de 2016 APROBADO: 17 de enero de 2017

CORRESPONDENCIA:

Alma Brenda Leyva Carreras, Universidad de Sonora, Departamento de Contabilidad, Blvd. Luis Encinas y Rosales, s/n Colonia Centro, C.P. 83000. Hermosillo, Sonora, México.

a Doctora, profesora-investigadora de tiempo completo en la Universidad de Sonora, México. Correo electrónico: almabrenda@eca.uson.mx

b Doctor, profesor-investigador de tiempo completo y jefe del Departamento de Economía en la Universidad de Sonora, México. Correo electrónico: jespejel@pitic.uson.mx

c Doctora, profesora-investigadora en la Universidad Popular Autónoma del Estado de Puebla, México. Correo electrónico: cavazosjuditho1@gmail.com 


\section{¿CÓMO CITO EL ARTÍCULO?} HOW TO CITE THIS PAPER?

CHICAGO:

Leyva-Carreras, Alma Brenda, Espejel-Blanco, Joel Enrique y Cavazos-Arroyo, Judith. 2017. "Habilidades gerenciales como estrategia de competitividad empresarial en las pequeñas y medianas empresas (Pymes)". Perspectiva Empresarial 4(1): 7-22. http://dx.doi. org/10.16967/rpe.v4n1a1

MLA:

Leyva-Carreras, Alma Brenda, Espejel-Blanco, Joel Enrique y Cavazos-Arroyo, Judith. "Habilidades gerenciales como estrategia de competitividad empresarial en las pequeñas y medianas empresas (Pymes)". Perspectiva Empresarial 4.1 (2017): 7-22. Digital. http:// dx.doi.org/10.16967/rpe. v4n1a1

\section{Managerial Skills as a Corporate Competitiveness Strategy in Small and Medium-Size Businesses}

ABSTRACT The purpose of this paper is to measure the impact of managerial skills as an internal factor in corporate competitiveness, as perceived by the entrepreneurs of small and medium-size businesses (SMEs) of Hermosillo, Sonora, Mexico. For such purpose, a mixed methodology was used in order to collect, analyze, and link quantitative and qualitative data, as well as to give an answer to the hypothesis of this research with a higher scientific evidence. Through an interview conducted on the experts, the first phase was an exploration based on the Delphi methodology in order to contextualize the problem to be addressed and validate the measurement instrument. In the second phase, 108 SMEs were sampled and a statistical analysis was conducted using PLS structural equation modelling to obtain results. The conclusions show that managerial skills are an internal factor of SMEs which have a significant impact on their corporate competitiveness.

KEYWORDS corporate competitiveness, manager, managerial skills, small and medium-size businesses.

\section{Habilidades gerenciais como estratégia de competitividade empresarial nas pequenas e medias empresas (PME)}

RESUMO Esta pesquisa tem como objetivo medir a influência das habilidades gerenciais como um fator interno na competitividade empresarial percebida por empresários de pequenas e médias empresas (PME) em Hermosillo, Sonora, México. Para isto, foi utilizada uma metodologia mista a fim de recolher, analisar e vincular os dados quantitativos e qualitativos e responder à abordagem de pesquisa com maior evidência científica. A primeira fase foi exploratória por meio de entrevistas ao painel de especialistas usando o método Delphi para dar contexto à problemática a analisar e avaliar o instrumento de medição. $\mathrm{Na}$ segunda fase foram amostrados 108 PME e foi realizada uma análise estatística usando equações estruturais (PLS) para obter resultados. As conclusões demonstraram que as habilidades gerenciais são um fator interno das PME que influenciam consideravelmente a sua competitividade empresarial.

PALAVRAS CHAVE competitividade empresarial, gerente, gestão, pequenas e médias empresas, habilidades gerenciais. 


\section{Introducción}

Hoy día, las organizaciones se encuentran en un contexto empresarial heterogéneo, caracterizado por hechos económicos como la globalización, la competitividad, las nuevas tecnologías y la proliferación de entornos complejos e impredecibles. Un contexto de cambio continuo, que obliga a las organizaciones a adaptarse para competir, tanto en el mercado internacional, como en los mercados internos (Gómez, 2008).

Lo anterior crea una dinámica a nivel mundial que presiona a las organizaciones a ser competitivas o dejar de existir. En este sentido, la competitividad empresarial se ha convertido en una exigencia para la supervivencia de las empresas (Artail, 2007). La comprensión de esta circunstancia es particularmente importante para las Pymes, que por sus características estructurales suelen encontrarse en desventaja con respecto a la gran empresa, la cual tiene a su disposición mayores recursos y capacidades (AECA, 2002).

Ante esta situación, la actual dinámica de los mercados exige un desarrollo de los sectores productivos, donde las competencias gerenciales se convierten en fuente de estrategia competitiva, impulsando la toma de decisiones y potenciando la diferenciación en el mercado (De Meuse, Dai \& Wu, 2011).

La necesidad de hacer bien nuevas cosas y en forma proactiva se convierte en el gran reto para la empresa que compite en ese mercado global. Ya no se trata de mantener ventaja en el pasado; es necesario generar resultados que pongan a la organización con ventaja en un espacio incierto (Eisenman, 2012). En tal sentido, se presume que la gerencia de una organización puede, a través de estrategias competitivas, desarrollar una gran gama de alternativas que le permitan aprovechar sus fortalezas y oportunidades para generar ventajas competitivas (Spendlove, 2007).

Con esta investigación se pretende analizar la importancia de un recurso estratégico para la organización: las habilidades gerenciales como elemento de las competencias que se consideran una fuente valiosa para la creación de valor y el desarrollo de la capacidad para competir en las organizaciones actuales, que intentan liderar el mercado y alcanzar un proceso de creación continua y de competitividad empresarial. El trabajo tiene como objetivo medir la influencia de las habilidades gerenciales como factor interno en la competitividad empresarial que perciben los empresarios de las Pymes de Hermosillo, Sonora, México, por medio de un modelo estadístico causal propuesto. A lo largo de cuatro secciones adicionales, se presentará el marco conceptual, así como la metodología que se siguió para realizar la investigación; además, se indicará cuál ha sido el proceso de análisis de los datos y los resultados, al igual que las conclusiones y algunas recomendaciones e implicaciones sociales.

\section{Marco teórico-conceptual y formulación de hipótesis}

\section{Revisión de la literatura}

La estrategia es una herramienta gerencial vital para determinar la orientación de la empresa, de tal forma que facilite la transformación y adecuación de la organización a las realidades del entorno competitivo (Hudson \& Smith, 2007). Autores como Garrido (2003) y Thompson \& Strickland (2004) consideran la estrategia como un proceso administrativo de entender a la organización, para visualizar su futuro a través de la innovación y la creatividad, lo cual implica la utilización de instrumentos conceptuales y herramientas prácticas que conlleven a crear ventajas para el éxito y seguir de cerca su entorno.

Resulta interesante incorporar una visión filosófica de gestión basada en la estrategia. Según Romero (2001), Dess \& Lumpkin (2003), Garrido (2003) y Thompson \& Strickland (2004), si se integra visión, misión, objetivos, principios, valores, estrategias y formas de planificación, que posea cualquier empresa, la conjunción de dichos elementos da como resultado una filosofía que otorga dinamismo a la organización. Puede afirmarse que esa filosofía imprime una cualidad para sobrevivir y progresar en presencia de una estrategia si se define la competitividad como intrínseca de la capacidad organizativa (Antonorsi, 2004), para precisar, así, la actuación gerencial.

Con el fin de centrarse en el concepto de estrategia como fuente de ventaja competitiva, Lloyd-Reason \& Mughan (2002) sugieren que los gerentes deben asumir conductas sobre pensamiento conceptual, visto como una herramienta esencial para administrar y entender a la organización; visión estratégica, para entender la influencia del entorno en la organización, y sobre 
todo habilidades gerenciales para la toma de decisiones asertivas, con base en la información y las condiciones del entorno.

En la actualidad, las habilidades personales, interpersonales y directivas para la gestión están adquiriendo cada vez mayor importancia en las empresas y organizaciones. En este sentido, Goyal (2013) afirma que las personas con excelentes habilidades personales llegan a la cima de su eficacia personal y de su organización, puesto que las habilidades interpersonales permiten a los trabajadores interactuar con éxito en un trabajo cada vez más desafiante sometido a un entorno cambiante.

Agolla \& Van Lill (2013) plantean que los principales factores internos de las innovaciones de la organización son la estrategia organizacional, el clima organizacional, el liderazgo estratégico, la capacidad empresarial y los recursos intangibles. Ciertamente, la motivación, el liderazgo, la toma de decisiones, la delegación y el manejo de los conflictos son algunas de las habilidades interpersonales que la gerencia eficaz va a tener que llevar a la práctica en el ejercicio de su gestión.

Zahra, Neubaum \& Naldi (2007) mencionan que el mundo cambiante de hoy requiere gerentes con habilidades indispensables para lograr resultados competitivos: excelentes interrelaciones con sus colaboradores, proveedores, clientes, $\mathrm{y}$ todos aquellos que intervienen en la cadena de valor. Adicional a las destrezas y aptitudes, el actual gerente debe poseer conocimientos prácticos en temas económicos, financieros, comerciales, jurídicos, de mercadeo, de gestión humana, y dominar dos o más idiomas.

Mitchelmore \& Rowley (2013) integran los modelos anteriores de competencias empresariales hacia el desarrollo de un marco de competencia empresarial, y hacen hincapié en que la gerencia es factor clave para la competencia empresarial y debe contar con los siguientes elementos: competencias emprendedoras, negocios y gestión por competencias.

Tonidandel, Braddy \& Fleenor (2012) realizaron un estudio con el propósito de examinar la importancia relativa de las cuatro dimensiones de habilidades gerenciales (habilidad técnica, habilidad administrativa, habilidad humana y comportamiento de la ciudadanía) para predecir la eficacia de la gestión. Además, su objetivo es explorar si la importancia relativa de estas dimensiones de habilidad varía en función del sexo o los niveles de jerarquía organizacional. Los investigadores encontraron que solamente el nivel jerárquico es un moderador significativo de la relación de habilidad-eficacia.

Por su parte, Koenigsfeld, Youn, Perdue \& Woods (2012) clasifican las competencias gerenciales en cinco dominios: el dominio conceptual-creativo, la dirección dominio, el dominio administrativo, el dominio interpersonal y el dominio técnico. El trabajo en equipo, la comunicación, la coordinación, la ejecución y el aprendizaje continuo son competencias críticas para el éxito de los mandos de gerencias medias (Xuejun Qiao \& Wang, 2009).

Los cambios que sufren las organizaciones afectan sin duda alguna el operar de los funcionarios, por lo que surge la necesidad de asegurar la mejor contribución de estos a los resultados esperados por la entidad. De allí que los gerentes deben tener la capacidad de obtener lo mejor de los trabajadores, propiciando al mismo tiempo la satisfacción profesional, por lo que se hace cada día más imprescindible que todos los gerentes estén dispuestos a asumir estos desafíos, y ello implica tener un manejo de las competencias básicas y esenciales en lo que respecta a su labor dentro de las organizaciones (Spendlove, 2007).

\section{La influencia de las habilidades gerenciales sobre la competitividad empresarial de las Pymes}

Las habilidades gerenciales, según Longenecker, Moore, Petty \& Palich (2009), están conformadas por el conjunto de conocimientos, habilidades, destrezas, comportamientos y aptitudes que necesita una persona para ser eficiente en una amplia gama de labores gerenciales y en diversas organizaciones. Por otra parte, no se puede desconocer que las personas recurren en su vida cotidiana a diversas competencias, incluidas las necesarias para ser eficientes en las relaciones personales, en actividades recreativas, en actividades laborales. En esta oportunidad se centrará la atención en las competencias gerenciales y su relación con la competitividad empresarial.

Al respecto, es relevante señalar que estas competencias son variadas, por cuanto se puede contemplar la conducta desde múltiples puntos de vista y llegar a definiciones cuyos matices son heterogéneos. La gerencia por competencias implica la adopción de un enfoque que supere el tradicional enfoque del rasgo de personalidad, lo que se fundamenta en una serie de importantes 
ventajas, tanto para la organización como para la persona (Bateman \& Snell, 2005).

Diversos estudios como los realizados por Samujh \& El-Kafafi (2010), Barhem, Younies \& Smith (2011), Tonidandel et al. (2012) y Kramar \& Steane (2012) detallan y clasifican en sus conclusiones la importancia del desarrollo de competencias gerenciales como un imperativo estratégico del negocio. Por tanto, las habilidades de la gerencia son clave para una gestión eficaz de la Innovación + Desarrollo (I + D), donde la capacidad técnica no es suficiente para ser un gerente eficaz de la I + D. Las diferencias de estilos de aprendizaje son primordiales en la adquisición de habilidades interpersonales, las cuales se pueden encaminar para desarrollar competencias de liderazgo que ayuden a crear estrategias de competitividad empresarial (ver por ejemplo, Dreyfus, 2008; De Meuse et al., 2011; Koenigsfeld et al., 2012; Thorn, 2012; Tonidandel et al., 2012; Zhang, Zuo \& Zillante, 2013).

Basado en lo anterior, el modelo cuenta con cinco habilidades gerenciales:

1. Operativa y de gestión: habilidad de la gerencia para disponer de un modelo de gestión compuesto por un conjunto de tareas y procesos enfocados a la mejora de las organizaciones internas, con el fin de aumentar su capacidad para conseguir los propósitos de sus políticas y sus diferentes objetivos operativos,

2. Administración estratégica: la gerencia debe tener la habilidad para crear el proceso de formulación e implementación de acciones, que mediante el análisis y el diagnóstico tanto del ambiente externo como del ambiente interno de la organización, le permita destacar las ventajas competitivas, e igualmente pueda aprovechar las oportunidades o defenderse de las amenazas que el ambiente le presenta a la organización en orden de conseguir sus objetivos declarados.

3. Planeación estratégica: habilidad de la gerencia para elaborar, desarrollar y poner en marcha los distintos planes operativos por parte de la empresa, con la intención de alcanzar objetivos y metas planteadas, ya sea a corto o a largo plazo.

4. Globalización: habilidad para tomar decisiones adecuadas con alto grado de confianza, que le permitan desarrollar una alta capacidad de negociación, análisis financiero y amplio conocimiento de metodologías para evaluación de proyectos, proceso y productos.

5. Recursos humanos: habilidad para identificar el valor del recurso humano y crear estrategias para potenciarlo como un activo intangible que tiene la capacidad de apoyar el incremento de la productividad, impulsar la innovación y con ello la competitividad.

En el presente trabajo se adoptó el concepto de competitividad dado por Aragón \& Rubio (2006), quienes la definen como la capacidad de una empresa para conseguir alcanzar una posición competitiva favorable que permita obtener un desempeño superior al de los competidores.

En este sentido, se consideran las siguientes hipótesis de trabajo basadas en las habilidades consideradas en el modelo propuesto para la creación de perfil por competencias gerenciales:

$\mathbf{H}_{\mathbf{1}}$ Las habilidades de competencias gerenciales, medidas a través de la variable operativa y de gestión, afectan directa y positivamente a la competitividad empresarial.

$\mathbf{H}_{2:}$ La habilidad de competencia gerencial, medida a través de la administración estratégica, tiene un efecto directo y positivo sobre la competitividad empresarial.

$\mathbf{H}_{3:}$ La habilidad de competencia gerencial, medida a través de la planeación estratégica, tiene un efecto directo y positivo sobre la competitividad empresarial.

$\mathbf{H}_{\text {4: }}$ La habilidad de competencia gerencial, medida a través de la visión de globalización, afecta directa y positivamente la competitividad empresarial.

$\mathbf{H}_{5:}$ La habilidad de competencia gerencial, medida a través de la variable de recursos humanos, afecta directa y positivamente la competitividad empresarial.

Planteadas las hipótesis de trabajo, el modelo causal propuesto quedaría reflejado tal y como se muestra en la figura 1. En este se establecen la influencia de las habilidades gerenciales sobre la competitividad empresarial manifestada en el comparativo de sustento teórico y la argumentación exploratoria realizada mediante panel de expertos. 
FIGURA 1. Modelo conceptual. Habilidades de competencias gerenciales para la competitividad empresarial

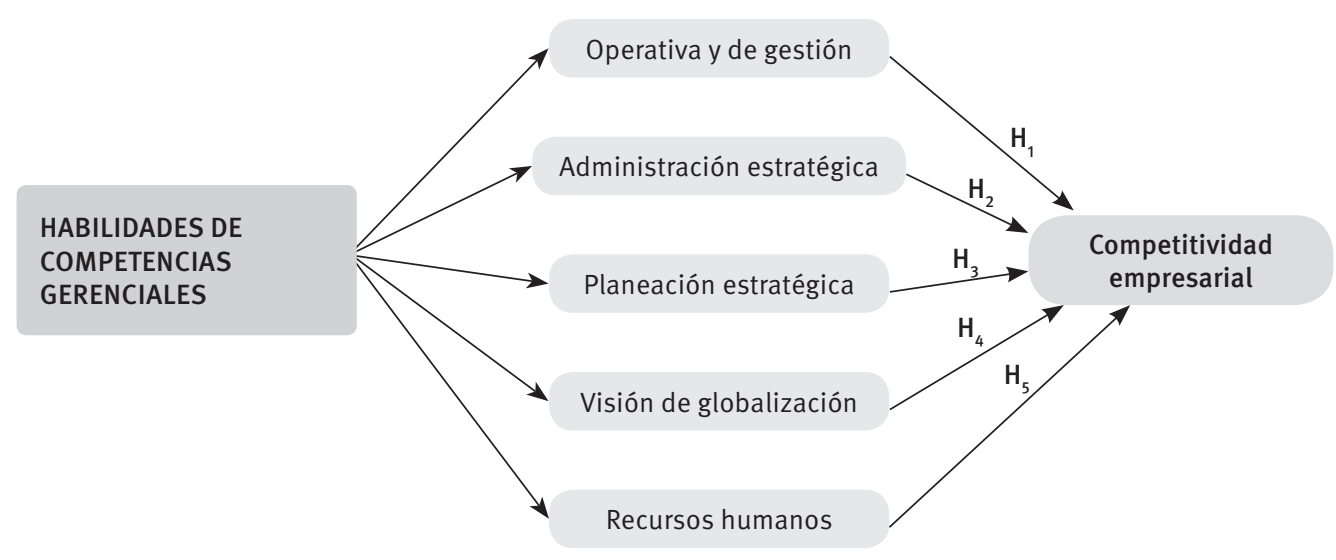

\section{Metodología}

Con el propósito de contrastar las hipótesis formuladas se desarrolla una serie de actuaciones realizadas bajo dos fases de una investigación mixta. En una primera fase cualitativa se llevó a cabo un análisis exploratorio apoyado en un panel de expertos (conocido también como método Delphi) con el propósito de validar el instrumento de medida (validez aparente). El panel fue integrado por seis expertos pertenecientes a tres sectores relacionados con las Pymes: gobierno, cámara y educación, los cuales se eligieron en función del objetivo prefijado y atendiendo a criterios de experiencia, posición, responsabilidad, acceso a la información, disponibilidad, y que tuvieran características relacionadas con Pymes. En este contexto, cabe destacar la preponderancia del modelo denominado de triple hélice (Mejía, 2004; González, 2009): universidad, industria, gobierno.

Así como la primera fase, dicho en forma resumida, es un panel de discusión abierto, la segunda fase es la encuesta a expertos con la ayuda de un cuestionario semiestructurado. El cuestionario tiene como objetivo que, con base en la opinión de los expertos, se pueda identificar la influencia de las habilidades gerenciales como factor interno de competitividad empresarial de las Pymes de Hermosillo, Sonora, México.

Los integrantes de la investigación recopilaron la información utilizando como soporte un cuestionario estructurado mediante entrevistas personales. Las entrevistas fueron dirigidas a los gerentes de las Pymes afiliadas tanto a la Cámara Nacional de Comercio, Servicios y Turismo (Canaco-Servytur) ${ }^{1}$ como a la Cámara Nacional de la Industria de Transformación (Canacintra)², con sede en Hermosillo, Sonora, México.

El tamaño de la muestra (108 cuestionarios válidos; ver tabla 1), se obtuvo una vez llevado a cabo el proceso de depuración. En este proyecto y a conveniencia de la investigación se utilizó una fórmula para una población finita y un muestreo sin reemplazo:

$$
\mathrm{n}=\frac{\mathrm{NZ} \mathrm{Z}^{2} \mathrm{pq}}{\left(\mathrm{Z}^{2} \mathrm{pq}\right)+\left[\mathrm{d}^{2}(\mathrm{~N}-1)\right]}
$$

1 Organismo que representa, defiende y promueve la actividad empresarial del comercio, los servicios y el turismo del Estado, brindando asesoría y atención de calidad que cubran las necesidades del empresario, impulsando el desarrollo de la sociedad y nuestra región. http://www.canacohermosillo.com.mx

2 Organismo que representa y defiende los intereses generales de la industria de transformación, y es un órgano de consulta y colaboración en el diseño y ejecución de políticas, programas e instrumentos que faciliten la expansión de la actividad económica y promotora de las actividades y desarrollo de sus empresas afiliadas. http://www.canacintrahermosillo.com/ 
TABLA 1. Ficha técnica

\begin{tabular}{ll}
\hline ÁMBITO GEOGRÁFICO & Hermosillo, Sonora \\
\hline UNIVERSO & 538 empresarios \\
\hline UNIDAD MUESTRAL & $\begin{array}{l}\text { Pymes afiliadas a Canaco-Servytur } \\
\text { y Canacintra }\end{array}$ \\
\hline METODOLOGÍA & $\begin{array}{l}\text { Encuesta con cuestionario } \\
\text { semiestructurado }\end{array}$ \\
\hline PROCEDIMIENTO & Muestra finita sin reemplazo \\
DE MUESTREO & 108 encuestas válidas \\
\hline TAMAÑO MUESTRAL & $\pm 5,0 \%$ \\
\hline ERROR MUESTRAL & $90 \% ;$ pq $=0,5$ \\
\hline NIVEL DE CONFIANZA & Agosto - Diciembre de 2015 \\
\hline FECHA DEL TRABAJO & \\
DE CAMPO &
\end{tabular}

Fuente: elaboración propia, 2015.

La información se obtuvo a través de las respuestas a un cuestionario que contenía preguntas cerradas. Los entrevistados tenían que mostrar su nivel de acuerdo o desacuerdo con una serie de afirmaciones utilizando una escala de medida de Likert de 7 puntos. Específicamente, la información brindada por el gerente se refería, por una parte, a la influencia de las habilidades gerenciales como factor interno de competitividad empresarial de las Pymes de Hermosillo, y por otra, a las características sociodemográficas del empresario.

\section{Análisis estadístico de los datos}

Para hacer el análisis estadístico de los datos se utilizó el método de modelos de ecuaciones estructurales (SEM, por sus siglas en inglés) con la técnica de mínimos cuadrados parciales (PLS, por sus siglas en inglés), aplicando el paquete informático estadístico SmartPLS 2.0 (Ringle, Wende \& Will, 2005). En los siguientes apartados se comprueba cada uno de los criterios estadísticos propuesto por la técnica PLS en referencia a la validez del modelo causal propuesto.

\section{Validación del modelo de medida}

Para validar el modelo de medida se llevaron a cabo las actuaciones metodológicas que se describen a continuación.

\section{Validez de contenido de las escalas de medida}

Para ello se revisó exhaustivamente la literatura especializada en habilidades gerenciales y competitividad empresarial con el objetivo de sustentar teóricamente los constructos y sus indicadores.

\section{Validez aparente de las escalas de medida}

Se comprobó que cada indicador de medida reflejara realmente aquello que se pretendía medir. Dichos enunciados se adaptaron y matizaron tras los estudios exploratorios previos, y luego el instrumento estadístico se sometió a un panel de expertos en el tema objeto de análisis, por medio de la técnica cualitativa del método Delphi, para depurar lo que no mostraba relación con la investigación; eso permitió a los autores de este documento garantizar la obtención de resultados satisfactorios, tal y como propone Zaichkowsky (1985).

\section{Validez individual de los indicadores}

Para este procedimiento estadístico se comprobó la fiabilidad individual de los indicadores reflectivos, los cuales deben poseer una carga factorial $(\lambda)$ igual o superior que 0,707 para constructos teóricos fuertes (Carmines \& Zeller, 1979) y de igual o superior a 0,550 para modelos teóricos flexibles (Hair, Anderson, Tatham \& Black, 1999). Cabe tener presente que las cargas factoriales que muestran que la varianza compartida entre el constructo y sus respectivos indicadores sean mayores que la varianza del error en un 50 por ciento. Considerando el criterio de aceptación anterior $(\lambda \geq 0,550)$, se depuraron los indicadores reflectivos que no cumplieron con la regla estadística (ver tabla 2). También se calculó la parte de la varianza que es explicada por el constructo (Bollen, 1989), por medio de la comunalidad $\left(\lambda_{i}^{2}\right)$ de las variables manifiestas. Una vez depurados los indicadores, se precedió a la nueva estimación del modelo de medida, con el fin de establecer la comunalidad de los mismos. Para determinar el procedimiento estadístico anterior se estimó el cuadrado de la correlación entre las variables manifiestas y su propia variable latente. Por ejemplo, para el primer indicador OG3 se tiene una carga factorial de $\lambda=0,661$, que representa una comunalidad de $\lambda^{2}=0,4369$, lo que indica que el $43,69 \%$ de la varianza de la variable manifiesta está relacionada con el constructo habilidades en el perfil por competencias gerenciales. 
TABLA 2. Fiabilidad individual de los indicadores

\begin{tabular}{|c|c|}
\hline INDICADORES & FIABILIDAD INDIVIDUAL \\
\hline $\begin{array}{l}\text { OG3: "Capacidad que trascender de lo lógico a lo abstracto y encontrar las verdaderas } \\
\text { causas y soluciones de una situación o problema específico, cuyos resultados son } \\
\text { sustentados con un alto nivel de eficacia y confiabilidad". }\end{array}$ & $\left(\lambda=0,661 ; \lambda^{2}=0,4369\right)$ \\
\hline OG4: “Capacidad para analizar, organizar y presentar datos numéricos de manera exacta". & $\left(\lambda=0,659 ; \lambda^{2}=0,4342\right)$ \\
\hline $\begin{array}{l}\text { OG5: "Habilidad para el manejo de técnicas mediante las cuales es posible formular } \\
\text { problemas de tal forma que puedan resolverse usando operaciones aritméticas". }\end{array}$ & $\left(\lambda=0,618 ; \lambda^{2}=0,3819\right)$ \\
\hline OG6: "Habilidad para comunicarse de forma oral y escrita con otras personas". & $\left(\lambda=0,696 ; \lambda^{2}=0,4844\right)$ \\
\hline $\begin{array}{l}\text { OG7: “Habilidad para expresarse eficazmente dando muestras de manejar un lenguaje } \\
\text { técnico y profesional con un tono muy espontáneo, acorde a su nivel de formación y } \\
\text { experiencia, así como del nivel del cargo que ocupe, lo que incide directamente en el nivel } \\
\text { de impacto personal del trabajador”. }\end{array}$ & $\left(\lambda=0,693 ; \lambda^{2}=0,4802\right)$ \\
\hline $\begin{array}{l}\text { OG8: "Excede las expectativas del cliente demostrando un compromiso total en la } \\
\text { identificación de cualquier problema y proporcionando las soluciones efectivas para la } \\
\text { solución del mismo". }\end{array}$ & $\left(\lambda=0,697 ; \lambda^{2}=0,4858\right)$ \\
\hline OG12: "Habilidad para llegar a acuerdos satisfactorios para todos". & $\left(\lambda=0,681 ; \lambda^{2}=0,4637\right)$ \\
\hline $\begin{array}{l}\text { PE18: "Capacidad para mejorar el comportamiento medioambiental de las actividades de } \\
\text { la organización". }\end{array}$ & $\left(\lambda=0,692 ; \lambda^{2}=0,4788\right)$ \\
\hline GL8: “Amplios conocimientos del comercio internacional”. & $\left(\lambda=0,594 ; \lambda^{2}=0,3528\right)$ \\
\hline $\begin{array}{l}\text { GL9: “Habilidad para planificación y desarrollo de estrategias comerciales, detectar } \\
\text { oportunidades comerciales y de nuevos mercados en el exterior para fijar los precios y } \\
\text { establecer políticas de cobranza”. }\end{array}$ & $\left(\lambda=0,648 ; \lambda^{2}=0,4199\right)$ \\
\hline
\end{tabular}

Fuente: elaboración propia con base en los datos recopilados, 2015.

\section{Validez de los constructos por medio de la convergente y divergencia estadística}

En aras de determinar esta prueba estadística, se analizó la fiabilidad del constructo a través de la medida del coeficiente Alfa de Cronbach $(\alpha)$ y el coeficiente de fiabilidad compuesta del constructo $\left(\rho_{c}\right)$, con el objetivo de demostrar la consistencia interna de los indicadores que miden los constructos reflectivos. A pesar de que 0,700 es un valor aceptable para el Alfa de Cronbach y para la confiabilidad compuesta en las primeras etapas de la investigación, para etapas más avanzadas los valores aceptables están entre 0,800 y 0,900 . Valores de 0,600 o menos indican falta de confiabilidad (Henseler, Ringle \& Sinkovics, 2009, p. 299). En la tabla 3 se muestra que el Alfa de Cronbach es mayor que 0,700 en todos los casos (Nunnally, 1978; Sanz, Ruiz \& Aldás, 2008). En cuanto al coeficiente de fiabilidad compuesta o coeficiente Rho de Spearman, todos los constructos reflectivos tienen valores mayores que 0,600 (Bagozzi \& Yi, 1988; Chin, 1998; Steenkamp \& Geyskens, 2006). Así mismo, se verificó que todos los coeficientes de fiabilidad compuesta hubieran sido superiores a los valores de alfa de Cronbach de 0,70 , para cada uno de los constructos propuestos (Fornell \& Larcker, 1981). De la misma manera, el coeficiente del análisis de la varianza extraída (AVE) debe ser mayor que 0,500 (Bagozzi, 1981; Fornell \& Lacker, 1981) y significativa al nivel de 0,01 (Sanzo, Santos, Vázquez \& Álvarez, 2003), lo cual indica que más del $50 \%$ de la varianza del constructo se debe a sus indicadores. Por tanto, se podría decir que los constructos propuestos en el modelo poseen una consistencia interna satisfactoria (ver tabla 3 ).

Para valorar la validez de constructo se realizaron dos análisis fundamentales:

1. La validez convergente (Fornell \& Larcker, 1981), se ha calculado mediante el análisis de la varianza extraída (AVE) para los constructos reflectivos. El coeficiente AVE proporciona la cantidad de varianza que un constructo reflectivo obtiene de sus indicadores en relación con la cantidad de varianza debida al error de medida. Como se muestra en la tabla 3, el coeficiente AVE para los constructos con indicadores reflectivos debe ser mayor que 0,500 (Bagozzi, 1981; Fornell \& Larcker, 1981), lo que indica que más del $50 \%$ de la varianza del constructo se debe a sus indicadores de medida. El análisis previo permite demostrar la validez convergente del modelo propuesto. 
TABLA 3. Fiabilidad de los constructos

\begin{tabular}{|c|c|c|c|}
\hline CONSTRUCTO INDICADORES & $\begin{array}{l}\text { ALFA DE } \\
\text { CRONBACH } \\
\text { (a) }\end{array}$ & $\begin{array}{l}\text { FIABILIDAD } \\
\text { COMPUESTA } \\
\left(\rho_{c}\right)\end{array}$ & $\begin{array}{l}\text { ANÁLISIS VARIANZA } \\
\text { EXTRAIIDA } \\
\text { (AVE) }\end{array}$ \\
\hline $\begin{array}{l}\text { Operativa y de gestión } \\
\text { (OG1; OG2; OG9; OG10; OG11) }\end{array}$ & 0,8483 & 0,8916 & 0,6223 \\
\hline $\begin{array}{l}\text { Administración estratégica } \\
\left(A_{1} ; A E_{2} ; A E_{3} ; A E_{4} ; A E_{5} ; A E_{6} ; A E_{7} ; A E_{8} ; A E_{9} ; A E_{10} ; A E_{11} ; A E_{12} ;\right. \\
\left.A E_{13} ; A E_{14} ; A E_{15} ; A E_{16}\right)\end{array}$ & 0,9667 & 0,9699 & 0,6683 \\
\hline $\begin{array}{l}\text { Planeación estratégica } \\
\text { (PE1; PE2; PE3; PE4; PE5; PE6; PE7; PE8; PE9; PE10; PE11; PE12; } \\
\text { PE13; PE14; PE15; PE16; PE17) }\end{array}$ & 0,9708 & 0,9734 & 0,6834 \\
\hline $\begin{array}{l}\text { Globalización } \\
\text { (GL1; GL2; GL3; GL4; GL5; GL6; GL7; GL10; GL11; GL12; GL13; } \\
\text { GL14) }\end{array}$ & 0,9509 & 0,9572 & 0,6516 \\
\hline $\begin{array}{l}\text { Recursos humanos } \\
\left(\mathrm{RH}_{1} ; \mathrm{RH}_{2} ; \mathrm{RH}_{3} ; \mathrm{RH}_{4} ; \mathrm{RH}_{5} ; \mathrm{RH}_{6} ; \mathrm{RH}_{7} ; \mathrm{RH}_{8} ; \mathrm{RH}_{9} ; \mathrm{RH}_{10} ; \mathrm{RH}_{11} ;\right. \\
\left.\mathrm{RH} 12 ; \mathrm{RH}_{13} ; \mathrm{RH}_{14} ; \mathrm{RH}_{15} ; \mathrm{RH}_{16}\right)\end{array}$ & 0,9734 & 0,9758 & 0,7167 \\
\hline 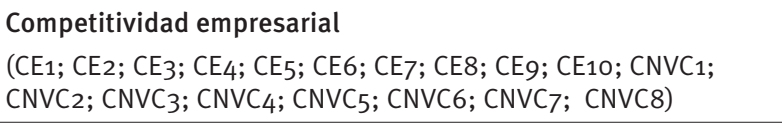 & 0,9726 & 0,9749 & 0,6839 \\
\hline
\end{tabular}

Fuente: elaboración propia con base en el análisis estadístico en SmartPLS 3.o.

2. La validez discriminante determina si el constructo propuesto está significativamente retirado de otros constructos con los que se encuentra relacionado teóricamente (Roldán, 2000). En este sentido, se examinaron los valores de la matriz de correlaciones entre constructos - que está formada por la raíz cuadrada del coeficiente AVE que deben ser superiores al resto de su misma columna-. Es decir, el coeficiente AVE de las variables latentes puede ser mayor que el cuadrado de las correlaciones entre las variables latentes, lo cual indica que cuanto mayor sea la porción de varianza entre los componentes de las variables latentes, mayores diferencias existirán entre los bloques de indicadores (Chin, 2000; Sánchez \& Roldán; 2005; Real, Leal \& Roldán, 2006). De acuerdo con Sánchez \& Roldán (2005), para que se cumpla la validez discriminante, los indicadores sobre la diagonal deben ser mayores que los indicadores por debajo de la diagonal (ver tabla 4). Como se observa en esa tabla, todos los indicadores cumplen con los criterios estadísticos propuestos en párrafos anteriores. Por tanto, se garantiza la validez discriminante de los distintos constructos que integran el modelo PLS propuesto.

\section{Validación del modelo estructural}

En cuanto a la validez del modelo estructural (Johnson, Herrmann \& Huber, 2006), se realizó el cálculo de las pruebas empíricas que se describen a continuación.

\section{Comprobar la varianza explicada (R2)}

Las variables endógenas o dependientes $\left(R^{2}\right)$ deben ser iguales o mayores que 0,100 (Falk \& Miller, 1992). A partir de este criterio empírico todos los constructos poseen un poder de predicción satisfactorio para el modelo estructural propuesto (tabla 5); todas las relaciones causales propuestas como hipótesis en los modelos de análisis cumplen con el criterio de aceptación.

\section{Verificar los coeficientes de regresión estandarizados ( $\beta$ )}

Estos factores deben alcanzar al menos un valor de 0,2 para que se consideren significativos (Chin, 1998). Como se puede observar en la tabla 5 , las relaciones causales propuestas en las hipótesis 2, 3 y 5 no cumplen con el criterio empírico de aceptación. 
TABLA 4. Matriz de correlaciones estandarizadas entre las diferentes variables latentes

\begin{tabular}{|c|c|c|c|c|c|c|}
\hline CONSTRUCTO & 1 & 2 & 3 & 4 & 5 & 6 \\
\hline Administración estratégica (1) & 0,8174 & & & & & \\
\hline Competitividad empresarial (2) & 0,7753 & 0,8269 & & & & \\
\hline Globalización (3) & 0,8572 & 0,7807 & 0,8072 & & & \\
\hline Operativa y de gestión (4) & 0,8153 & 0,6772 & 0,777 & 0,7888 & & \\
\hline Planeación estratégica (5) & 0,907 & 0,7564 & 0,8836 & 0,8176 & 0,8266 & \\
\hline Recursos humanos (6) & 0,8671 & 0,7437 & 0,8525 & 0,724 & 0,8736 & 0,8465 \\
\hline \multicolumn{7}{|c|}{$\begin{array}{l}\text { Nota: Los indicadores sobre la diagonal (en negritas) representan los resultados de la raíz cuadrada del AVE entre los constructos y sus respectivas medidas. } \\
\text { Los indicadores por debajo de la diagonal son las correlaciones entre los constructos. De acuerdo con Sánchez \& Roldán (2005), para que se cumpla la validez } \\
\text { discriminante, los indicadores sobre la diagonal deben ser mayores que los indicadores por debajo de la misma. }\end{array}$} \\
\hline
\end{tabular}

TABLA 5. Resultados del análisis estadístico

\begin{tabular}{|c|c|c|c|}
\hline HIPÓTESIS & $\begin{array}{l}\text { SIGNO DE LA } \\
\text { HIPÓTESIS }\end{array}$ & $\begin{array}{l}\text { COEFICIENTES PATH } \\
\text { ESTANDARIZADOS (B) }\end{array}$ & $\begin{array}{l}\text { VALOR } T \\
\text { (BOOTSTRAP) }\end{array}$ \\
\hline $\mathrm{H}_{1}$ : Operativa y de gestión .... Competitividad empresarial & + & 0,041 & $0,2763^{\star \star \star}$ \\
\hline $\mathrm{H}_{2}:$ Administración estratégica $\cdots \cdot$ : Competitividad empresarial & + & 0,3174 & 1,5892 n.s. \\
\hline $\mathbf{H}_{3}:$ Planeación estratégica $\cdots \cdot$ ? Competitividad empresarial & + & $-0,0034$ & o,0181n.s. \\
\hline $\begin{array}{l}\mathrm{H}_{4} \text { : Competencias gerenciales a través de la variable } \\
\text { Globalización .... Competitividad empresarial }\end{array}$ & + & 0,3779 & $2,2185^{\star \star}$ \\
\hline $\mathbf{H}_{5}:$ Recursos humanos .... Competitividad empresarial & + & 0,1196 & o,6628 n.s. \\
\hline
\end{tabular}

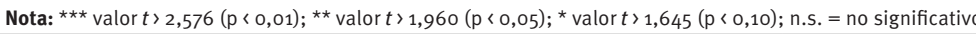

\begin{tabular}{|c|c|c|}
\hline CONSTRUCTO & VARIANZA EXPLICADA R² & PRUEBA DE STONE-GEISSER Q \\
\hline \multicolumn{3}{|l|}{ Administración estratégica } \\
\hline Competitividad empresarial & 0,6552 & 0,2661 \\
\hline \multicolumn{3}{|l|}{ Globalización } \\
\hline \multicolumn{3}{|l|}{ Operativa y de gestión } \\
\hline \multicolumn{3}{|l|}{ Planeación estratégica } \\
\hline \multicolumn{3}{|l|}{ Recursos humanos } \\
\hline
\end{tabular}

Fuente: elaboración propia con base en el análisis estadístico en SmartPLS 3.0

\section{Índice de bondad de ajuste (GoF)}

Finalmente, tras demostrar la validez del modelo, se calculó el índice de bondad de ajuste del modelo estructural, el cual fue de 0,6630 (ver tabla 6). Este resultado indica que se tiene un buen ajuste, que cumple con el criterio empírico de que la medida de bondad de ajuste debe variar entre 0 y 1; a mayor valor, mejor será el índice (Tenenhaus, 2008).

\section{Contraste de hipótesis de trabajo}

Una vez validados los modelos de medida y estructural, se analizaron los resultados obtenidos para contrastar las cinco hipótesis formuladas en el modelo propuesto. Lo anterior, con el fin de justificar y argumentar las posibles desviaciones de los resultados esperados.
Los resultados del modelo estructural ponen de manifiesto que la hipótesis 4: "la habilidad de competencia gerencial medida a través de la visión de globalización que perciben los gerentes de las Pymes de Hermosillo, Sonora, México afecta significativamente de forma directa y positiva sobre la competitividad empresarial (H4: $\beta=0,3779$; $\mathrm{p}<0,05$ ), tal y como lo evidencian los parámetros estadísticos. Por tanto, se encuentran indicios suficientes para aceptar la hipótesis H4. Sin embargo, no se encontró evidencia suficiente para aprobar las hipótesis $\mathbf{H 1}(\beta=0,041 ; \mathrm{p}<0,01)$ : "las habilidades de competencias gerenciales, medidas a través de la variable operativa y de gestión afectan directamente en la competitividad empresarial", H2: ( $\beta=0,3174$; n.s.): "la habilidad de competencia gerencial, medida a través de la administración estratégica, tiene un efecto directo y positivo sobre 
TABLA 6. Índice de bondad de ajuste (GoF)

\begin{tabular}{|c|c|c|c|}
\hline CONSTRUCTO & $\begin{array}{l}\text { ANÁLISIS VARIANZA EXTRAÍDA } \\
\text { (AVE) }\end{array}$ & $\begin{array}{l}\text { VARIANZA EXPLICADA } \\
\left(\mathrm{R}^{2}\right)\end{array}$ & $\begin{array}{l}\text { ÍNDICE DE BONDAD } \\
\text { DE AJUSTE } \underline{-}\end{array}$ \\
\hline Administración estratégica & 0,6683 & & \\
\hline Competitividad empresarial & 0,6839 & 0,6552 & \\
\hline Globalización & 0,6516 & & \\
\hline Operativa y de gestión & 0,6223 & & \\
\hline Planeación estratégica & 0,6834 & & \\
\hline Recursos humanos & 0,7167 & & \\
\hline Media aritmética & $\underline{0,6710}$ & $\underline{0,6552}$ & $\underline{0,6630^{a}}$ \\
\hline
\end{tabular}

a El índice de bondad de ajuste $(\mathrm{GoF})=\mathrm{V}(\mathrm{AVE})^{\star}\left(\mathrm{R}^{2}\right)$ (Tenenhaus et al., 2005; Esposito et al., 2008; Tenenhaus, 2008).

Fuente: elaboración propia con base en los resultados estadísticos en SmartPLS 3.0.

la competitividad empresarial", $\mathbf{H 3}(\beta=-0,0034$; n.s.): "la habilidad de competencia gerencial, medida a través de la planeación estratégica, tiene un efecto directo y positivo sobre la competitividad empresarial" y, H5: ( $\beta=0,1196$; n.s.): "la habilidad de competencia gerencial, medida a través de la variable recursos humanos, afecta directa y positivamente la competitividad empresarial". Estos resultados serán retomados para brindar una mayor explicación en la sección de discusión.

Así mismo, ante una mayor equidad relacional percibida por los gerentes de las Pymes de Hermosillo, Sonora, este hecho hace que se incrementen los niveles de habilidades para la mejora de las competencias gerenciales, dado que presenta el efecto esperado $\left(\mathrm{H}_{4}: \beta=0,3779 ; \mathrm{p}>0,01\right)$. Con ello se confirma que a mayores niveles de equidad relacional, se influye positiva y significativamente en la competitividad empresarial de las Pymes de Hermosillo, Sonora, México.

En la figura 2 se muestra el modelo conceptual y sus respectivas hipótesis de trabajo a partir de los modelos teóricos ya propuestos por la comunidad científica; lo anterior, para replicar y aportar nuevas relaciones causales al caso de las habilidades gerenciales en las Pymes de Hermosillo, Sonora, México.

FIGURA 2. Modelo final del desarrollo de competencias gerenciales para la competitividad empresarial de las Pymes de Hermosillo, Sonora
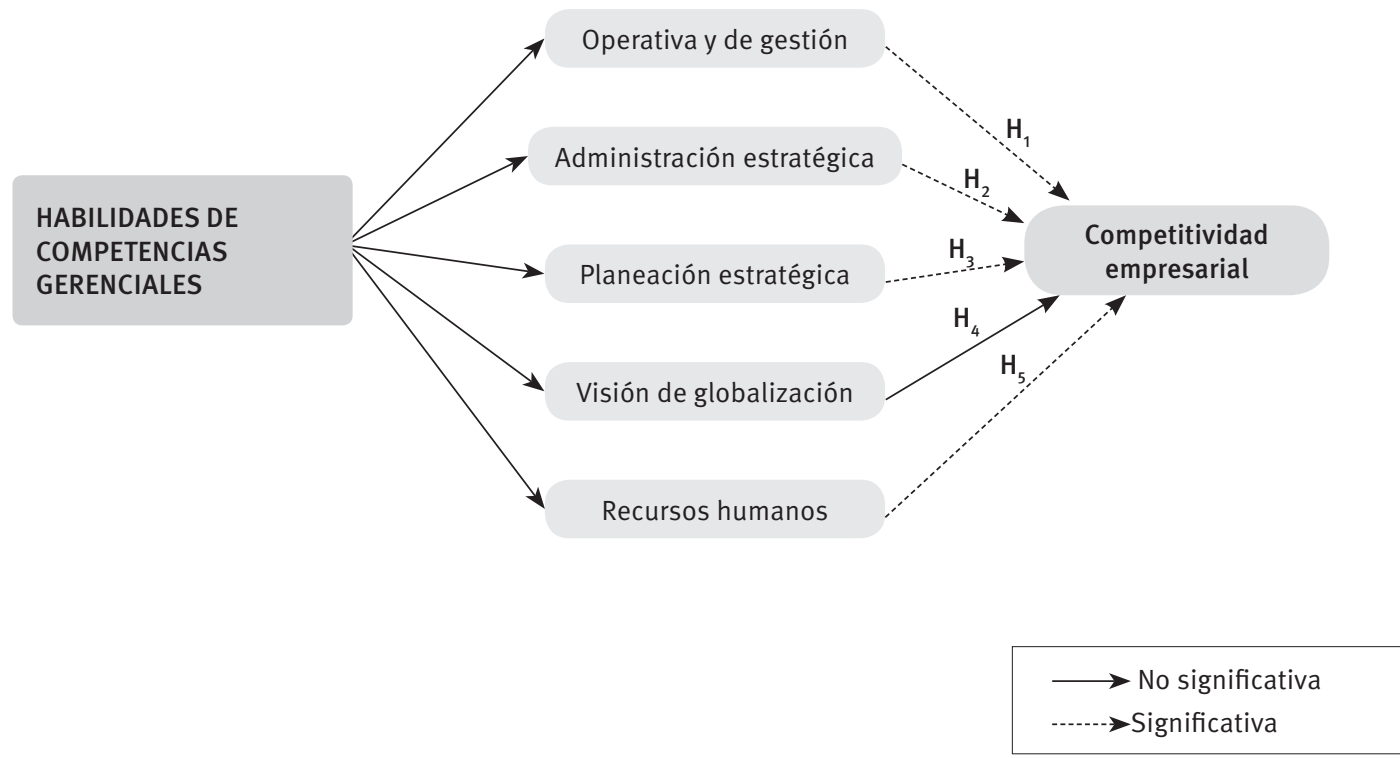


\section{Discusión e interpretación de resultados}

El método Delphi permitió contextualizar la problemática objeto de análisis, así como la validación del instrumento de medida. Para realizar este trabajo se revisó el modelo de triple hélice de relaciones entre universidad, industria y gobierno, ampliamente usado en los estudios de innovación (Mejía, 2004; González, 2009). Para esta investigación, la característica principal fue elegir expertos de tres sectores relacionados con Pymes, y se seleccionaron, eligiéndose los siguientes: dos del sector gobierno (representados por el secretario de economía del Estado de Sonora y el director de Nacional Financiera ${ }^{3}$ [NAFIN]), dos del sector industrial (representados por el presidente de la Cámara Nacional de Comercio, Servicios y Turismo (Canaco-Servytur) y el presidente de la Cámara Nacional de la Industria de Transformación (Canacintra), quienes son las cámaras con las que se realizó el trabajo, y, dos del sector educación (académicos que realizan proyectos con Pymes dentro de Instituciones de Educación Superior). El interés fundamental de la aplicación del estudio cualitativo (conocido también como método Delphi), se basa en las opiniones de un panel de expertos (conocido también como método Delphi), el cual se desarrolló conforme a lo señalado por Yáñez y Cuadra (2008) y; Ortega (2008). El estudio, se llevó a cabo en dos etapas: un panel de discusión abierto y, una encuesta a expertos con la ayuda de un cuestionario semiestructurado.

Los expertos opinaron que las habilidades gerenciales para la competitividad empresarial están por debajo de la media. Con el fin de formular estrategias que mantengan o incrementen una ventaja competitiva sustentable, los gerentes de las Pymes requieren desarrollar habilidades de análisis de la información. Las habilidades gerenciales otorgan esas capacidades mediante el trabajo que realiza el gerente para adquirir el desarrollo de estrategias empresariales que ayuden a lograr una ventaja competitiva dentro del mercado al que pertenece.

\footnotetext{
3 Una institución de banca de desarrollo en México encargada de promover el ahorro y la inversión a través de la gestión de proyectos, el establecimiento de programas de financiamiento, la coordinación de inversiones de capitales y el incremento de la productividad. http://www.nafin. com/portalnf/content/home/home.html
}

Una vez analizada la validación del modelo de medida, así como la del modelo estructural, se procedió a examinar los resultados obtenidos para contrastar las hipótesis de investigación formuladas, y con ello justificar y argumentar las posibles desviaciones de los resultados esperados.

La planeación estratégica y la administración estratégica son conceptos diferentes (Ansoff, Declerck, y Hayes, 1976) ya que trabajar con base en una misión, una visión y unos valores compartidos ayuda a definir la estrategia empresarial que sirve como base del trabajo de la planeación estratégica (Román, Arbeláez, y Patiño, 2012). Sin embargo, los resultados muestran que los empresarios perciben ambos conceptos como similares, pero tal como Martínez y Martínez (2009) aseveran, la validez de contenido actúa como un criterio robusto frente a determinados análisis estadísticos basados en covarianzas. Esto implica que aunque los sujetos de estudio confundan en la práctica los conceptos, la teoría permite diferenciarlos claramente.

Para el modelo propuesto, todos los indicadores cumplen con los criterios empíricos sugeridos a excepción de los constructos de Administración estratégica y Planeación estratégica. Estos constructos, a pesar de que son diferentes, de acuerdo con la teoría son estadísticamente similares en casi un 91\%. Martínez y Martínez (2009) aseveran que la validez de contenido actúa como criterio robusto frente a determinados análisis estadísticos basados en covarianzas.

Los resultados del modelo estructural ponen de manifiesto que las competencias gerenciales a través de la variable Globalización que perciben los gerentes de las Pymes de Hermosillo, Sonora, México, influyen de forma significativa sobre la competitividad empresarial $\left(\mathbf{H}_{4}: \beta=0,3779\right.$; $p<$ $0,05)$, posiblemente porque los gerentes están conscientes de las actuales tendencias económicas y de mercado, al igual que de la obligación que tienen de llevar a sus organizaciones a desarrollar una visión estratégica que les permita enfrentar los escenarios probables y tomar las decisiones más idóneas, de tal forma que se solventen los obstáculos que limitan el desempeño eficiente de sus empresas.

Se ha rechazado la hipótesis $\mathbf{H}_{1}(\beta=0,041 ; \mathrm{p}<$ $0,01)$ : "Las habilidades de competencias gerenciales, medidas a través de la variable operativa y de gestión, afectan directa y positivamente a la competitividad empresarial", posiblemente porque el empresario Pyme ejerce gestión empresarial de 
acuerdo con su formación y experiencia; utilizando sus propias estrategias hace uso de un lenguaje técnico y profesional con un tono espontáneo, y no se apoya en el análisis numérico para el análisis de problemas que le permitan ofrecer soluciones efectivas en los problemas suscitados con los trabajadores o clientes. Sin embargo, el entorno actual de la economía del país exige de la gerencia mayor innovación y participación para la búsqueda constante de estrategias que lleven a la empresa al desarrollo y crecimiento, para poder enfrentar la competitividad actual.

En el caso de la no significancia de la $\mathbf{H}_{2}:(\beta$ = 0,3174; n.s.): "La habilidad de competencia gerencial, medida a través de la administración estratégica, tiene un efecto directo y positivo sobre la competitividad empresarial", el resultado parece razonable porque la formación y ambición profesional del gerente hace que no tome en cuenta el macroentorno de su empresa. La falta de creatividad, espíritu emprendedor e innovación de parte de la gerencia hace que la toma de decisiones no sea efectiva, y hoy en día se requiere un gerente proactivo, donde la previsión sea una de sus características básicas para enfrentar el mundo cambiante de hoy.

En la $\mathbf{H}_{3}(\beta=-0,0034 ;$ n.s. $)$ : "La habilidad de competencia gerencial, medida a través de la planeación estratégica, tiene un efecto directo y positivo sobre la competitividad empresarial", la no significancia al parecer se da porque el gerente Pyme no desarrolla alternativas formales para mejorar el desempeño organizacional de sus empresas. El entorno competitivo de los negocios exige el éxito empresarial, para lo cual se exige a la gerencia analizar el contexto real al que se enfrenta la organización.

En el caso de la no significancia de la $\mathbf{H}_{5}:(\beta$ = 0,1196; n.s.): "La habilidad de competencia gerencial, medida a través de la variable de recursos humanos, afecta directa y positivamente la competitividad empresarial", el resultado parece razonable porque el gerente Pyme no considera relevante la sociabilidad y el trabajo en equipo de sus colaboradores, y además utiliza sus propios juicios y métodos de disciplina para poder medir el desempeño de sus empleados. Hablar de la importancia de los recursos humanos es discernir sobre los aportes que estos han brindado en el mundo actual, principalmente en el mundo de los negocios. En la actualidad, ser un puente entre las necesidades e intereses de una empresa y su personal ha contribuido significativamente a elevar el nivel de competitividad de cada organización que pretende estructurar y direccionar los esfuerzos de una serie de personas en torno al crecimiento de esta y al crecimiento profesional e incluso personal de cada individuo que la conforma.

\section{Conclusiones e implicaciones empresariales}

Los gerentes de las empresas de hoy deben ser capaces de combinar las capacidades de gestión, la creatividad y el espíritu empresarial dentro de una perspectiva capaz de abrazar al mismo tiempo el diseño y la producción, la tradición y la innovación, la realidad y la visión, la vida cotidiana y el futuro (Eisenmann, 2012).

Durante los resultados del primer modelo referente al impacto de las competencias gerenciales sobre la competitividad empresarial, se observa la caída de gran parte de los indicadores en el constructo de la competencia Operativa y de gestión; en el de la habilidad de Planeación estratégica solo se elimina uno, y dos en el de la habilidad de Globalización. En el resto de los constructos se observa que la totalidad de indicadores se conservaron, lo cual demuestra su importancia en la apreciación de los constructos de Recursos humanos y Competitividad empresarial, y exterioriza una mayor disposición de los gerentes Pymes para evaluar la competitividad empresarial de manera más objetiva, que se ve reflejado en la contundencia de los indicadores.

Los resultados estadísticos muestran que para el gerente Pyme, la administración estratégica y la planeación estratégica son similares. Sin embargo, Hitt, Duane \& Hoskisson (2008) mencionan que la administración estratégica es un estado de cultura que alcanza la empresa en todas sus áreas, y definen la planeación estratégica como un proceso que genera ventajas competitivas y da sustentabilidad de largo plazo a la empresa. Una empresa puede tener planeación estratégica y no administrarse estratégicamente, pero si tiene una cultura, la planeación estratégica se convierte en una responsabilidad y compromiso diario y natural de sus gerentes y directivos (French, Kelly \& Harrison, 2004). El empresario Pyme tiene que comprender que para lograr planeación estratégica es preciso establecer el hábito de crear, analizar y usar regularmente la información crítica para la toma de decisiones, generar pensamiento estratégico en el personal que toma decisiones, $\mathrm{y}$, sobre 
todo, fomentar y alentar un ambiente en el que el pensamiento diferente se convierta en vehículo que potencia la creatividad, la innovación y el desarrollo sustentable.

Los resultados obtenidos en esta investigación ponen de manifiesto la importancia que tiene para los empresarios Pymes de la ciudad de Hermosillo, Sonora, México, reconocer con un sustento científico la necesidad de buscar excelencia empresarial mediante el logro de ventajas sostenidas en el mercado, porque la excelencia está asociada con la competitividad empresarial, y para alcanzarla se requiere una gerencia dinámica, actualizada, con habilidades operativas y de gestión, administración estratégica, planeación estratégica, globalización y recursos humanos, siempre abierta al cambio organizativo y tecnológico.

Teniendo en cuenta este panorama, es necesario incluir medidas orientadas a la competitividad empresarial de las Pymes en el estado de Sonora, México. En primer lugar, hay que apoyar a las Pymes para que superen progresivamente deficiencias productivas a través de la mejora de gestión empresarial, ampliando sus opciones para enfrentar la competencia internacional, y una estrategia podría ser la adopción de competencias gerenciales. En segundo término, es preciso avanzar con iniciativas integradas que apunten a producir cambios estructurales dentro de estos negocios.

Lo anterior lleva a concluir, por tanto, que utilizar una planeación estratégica en el trabajo de gestión empresarial determinará las acciones que la gerencia deberá llevar a cabo para lograr el objetivo o la meta de la organización; es decir, le dará la ruta que se requiere seguir para la realización de sus actividades. Cuando no se tiene una planeación estratégica definida, el comportamiento en una empresa se tornará incongruente y en ocasiones hasta caótico, ya que no está delimitado cuál es el rumbo o qué es lo que se quiere lograr. El valor de la estrategia en una competencia proviene del desarrollo de la habilidad gerencial para intervenir en un sistema complejo con solo cierta información limitada, y con ello producir un cambio predecible y deseable en el equilibrio del sistema.

\section{REFERENCIAS}

Agolla, J. E., \& Van Lill, J. B. (2013). Public Sector Innovation Drivers. A Process Model. J Soc Science, 34(2), 165-176.
Ansoff, H. I., Declerck, R. P., \& Hayes, R. L. (1976). From strategic planning to strategic management. New York: John Wiley and Sons.

Antonorsi, M. (2004). Guía práctica de la empresa competitiva. Venezuela competitiva (3aㅡ. ed.). Caracas: Altolitho, C. A.

Aragón Sánchez, A., \& Rubio Bañón, A. (2006). Factores explicativos del éxito competitivo: El caso de las Pymes del estado de Veracruz. Revista Contaduría y Administración, 216, 63-85.

Artail, H. A. (2007). Application of KM measures to the impact of a specialized groupware system on corporate productivity and operations. Information \& Management, 43, 551-564.

Asociación Española de Contabilidad y Administración de Empresas, AECA. (2002). Factores determinantes de la eficacia y rentabilidad de la Pyme en España. Madrid: AECA.

Bagozzi, R. P. (1981). Evaluating structural equations models with unobservable variables and measurement error: a comment. Journal of Marketing Research, 18(3), 375-381.

Bagozzi, R. P., \& Yi, Y. (1988). On the evaluation of structural equation models. Journal of the Academy of Marketing Science, 16(1), 74-94.

Barhem, B., Younies, H., \& Smith, P. C. (2011). Ranking the future global manager characteristics and knowledge requirements according to UAE business managers' opinions. Education, Business and Society: Contemporary Middle Eastern Issues, 4(3), 229-247.

Bateman, S., \& Snell, S. (2005). Administración. Una ventaja competitiva (4⿳亠口䒑a. ed.). México: McGraw-Hill.

Bollen, K. (1989). Structural Equations with Latent Variables. New York: Wiley.

Carmines, E. G., \& Zeller, R. A. (1979). Reliability and Validity Assessment. Sage University Paper Series on Quantitative Applications in the Social Sciences, Series Nos. 07-017. Beverly Hills, CA: Sage.

Chin, W. W. (1998). The Partial Least Squares Approach to Structural Equation Modelling. En G. A. Marcoulides (Ed.). Modern Methods for Business Research. Mahwah, NJ: Lawrence Erlbaum Associates, Publisher.

Chin, W. W. (2000). Partial Least Square for researchers: An overview and presentation of recent advances using the PLS approach. Recuperado de http:// discnt.cba.uh.edu/chin/indx.html

De Meuse, K. P., Dai, G., \& Wu, J. (2011). Leadership competencies across organizational levels: a test of the pipeline model. Journal of Management Development, 30(4), 366-380.

Dess, L. \& Lumpkin, M. (2003). Dirección estratégica. Madrid: McGraw-Hill.

Dreyfus, C. R. (2008). Identifying competencies that predict effectiveness of R\&D Managers. Journal of Management Development, 27(1), 76-91. 
Eisenmann, T. (2012). Business Model Analysis for Entrepreneurs. Harvard Business School Publishing, 9, 1-25.

Esposito Vinzi, V., Trinchera, L., Squillacciotti, S., \& Tenenhaus, M. (2008). REBUS-PLS: A responsebased procedure for detecting unit segments in PLS path modelling. Applied Stochastic Models in Business and Industry, 24(5), 439-458.

Falk, R. F., \& Miller, N. B. (1992). A Primer for Soft Modeling. Akron: The University of Akron Press.

Fornell, C., \& Larcker, D. F. (1981). Evaluating structural equation models with unobservable variables and measurement error. Journal of Marketing Research, 18(1), 39-50.

French, S., Kelly, S., \& Harrison, F. (2004). The Role of Strategic Planning in the Performance of Small, Professional Service Firms. Journal of Management Development, 23, 765-776.

Garrido, S. (2003). Dirección estratégica. España: McGraw-Hill.

Gómez, H. M. (2008). Cultura organizacional e identidad productiva propuesta para el análisis de las organizaciones colombianas. Umbral Científico, 13, 56-64.

González, T. (2009). El modelo de triple hélice de relaciones universidad, industria y gobierno: un análisis crítico. ARBOR Ciencia, Pensamiento y Cultura, 738, 739-755.

Goyal, M. (2013). Importance of Interpersonal Skills at Workplace. International Indexed and Refereed Journal, 2013(V), 49.

Hair, J. F. Jr., Anderson, R. E., Tatham, R. I., \& Black, W. C. (1999). Análisis multivariante (5⿳a.. ed.). Madrid: Prentice Hall.

Henseler, J., Ringle, C. M., \& Sinkovics, R. R. (2009). The Use of Partial Least Squares Path Modeling in International Marketing. Advances in International Marketing, 20, 277-319.

Hitt, M. A., Duane Ireland, R., \& Hoskisson, R. E. (2008). Administración estratégica: competitividad y globalización. Conceptos y casos. México: Cengage Learning Latin America.

Hudson, M., \& Smith, D. (2007). Implementing strategically aligned performance measurement in small firms. International Journal Production Economics, 106, 393-408.

Johnson, M. D., Herrmann, A., \& Huber, F. (2006). The evolution of loyalty intentions. Journal of Marketing, 70(2), 122-132.

Koenigsfeld, J. P., Youn, H., Perdue, J., \& Woods, R. H. (2012). Revised competencies for private club managers. International Journal of Contemporary Hospitality Management, 24(7), 1066-1087.

Kramar, R., \& Steane, P. (2012). Emerging HRM skills in Australia. Asia-Pacific Journal of Business Administration, 4(2), 139-157.
Lloyd-Reason, L., \& Mughan, T. (2002). Strategies for internationalization within SMEs: key role of the owner-manager. Journal of Small Business and Enterprise Development, 9(2), 120-129.

Longenecker, J. C., Moore, C. W., Petty, J. W., \& Palich, L. E. (2009). Administración de pequeñas empresas: lanzamiento y crecimiento de iniciativas emprendedoras. México: Cengage Learning.

Martínez-García, J. A. \& Martínez-Caro, L. (2009). La validez discriminante como criterio de evaluación de escalas: ¿Teoría o estadística? University Psychol., 8(1), 27-36.

Mejía Navarrete, J. (2004). Sobre la investigación cualitativa. Nuevos conceptos y campos de desarrollo. Investigaciones Sociales, VII(13), 277-299.

Mitchelmore, S., \& Rowley, J. (2013). Growth and planning strategies within women-led SMEs. Management Decision, 51(1), 83-96.

Nunnally, J. C. (1978). Psychometric Theory. New York: McGraw-Hill.

Ortega, F. (2008). El método Delphi, prospectiva en Ciencias Sociales a través del análisis de un caso práctico. Revista Escuela de Administración de Negocios, 64, 31-54.

Real, J. C., Leal, A., \& Roldán, J. L. (2006). Information technology as a determinant of organizational learning and technological distinctive competencies. Industrial Marketing Management, 35(4), 505-521.

Ringle, C. M., Wende, S., \& Will, A. (2005). SmartPLS 2.0 (M3). Recuperado de http://www.smartpls.de

Roldán, J. L. (2000). Sistemas de información ejecutivos EIS. Génesis, implantación; repercusiones organizativas. (Tesis doctoral). Universidad de Sevilla.

Román, O. H., Arbeláez, G., y Patiño, C. A. (2012). Gerencia integral desde la perspectiva de un modelo de planeación estratégica. Gestión \& Desarrollo, 9(1), 51-78.

Romero, D. (2001). Presencia del pensamiento estratégico en la gran empresa del sector metalúrgico y metalmecánica. (Tesis doctoral). Universidad Dr. Rafael Belloso Chacín.

Samujh, R. H., \& El-Kafafi, S. (2010). Tool box for managers: lessons from New Zealand small businesses. World Journal of Enterpreneurship, Management and Sustainable Development, 6(1/2), 77-87.

Sánchez, M. J., \& Roldán, J. L. (2005). Web acceptance and usage model. A comparison between goal-directed and experiential web users. Internet Research, 15(1), 21-48.

Sanz, S., Ruiz, C. \& Aldás, J. (2008). La influencia de la dependencia del medio en el comercio electrónico B2C. Propuesta de un modelo integrador aplicado a la intención de compra futura en Internet. Cuadernos de Economía y Dirección de la Empresa, $36,45-76$. 
Sanzo, M., Santos, M., Vázquez, R., \& Álvarez, L. (2003). The Effect of Market Orientation on Buyer-seller Relationship Satisfaction. Industrial Marketing Management, 32(4), 327-345.

Spendlove, M. (2007). Competencies for effective leadership in higher education. International Journal of Educational Management, 21(5), 407-417.

Steenkamp, J. B. E. M. \& Geyskens, I. (2006). How country characteristics affect the perceived value of web sites. Journal of Marketing, 70(3), 136-150.

Tenenhaus, M., Vinzi, V. E., Chatelin, Y. M., \& Lauro, C. (2005). PLS path modeling. Computational statistics \& data analysis, 48(1), 159-205.

Tenenhaus, M. (2008). Structural Equation Modelling for small samples. Paris: Working Paper ${ }^{\circ}{ }^{\circ} 885$, HEC, Jouy-en-Josas.

Thompson, A. \& Strickland, A. (2004). Administración estratégica (13a. ed.). México: McGraw-Hill.

Thorn, I. M. (2012). Leadership in international organizations: Global leadership competencies. The Psychologist-Manager Journal, 15(3), 158-163.

Tonidandel, S., Braddy, P. W., \& Fleenor, J. W. (2012). Relative importance of managerial skills for predicting effectiveness. Journal of Managerial Psychology, 27(6), 636-655.

Xuejun Qiao, J., \& Wang, W. (2009). Managerial competencies for middle managers: some empirical findings from China. Journal of European Industrial Training, 33(1), 69-80.

Yáñez Gallardo, R. \& Cuadra Olmos, R. (2008). La técnica Delphi y la investigación en los servicios de salud. Ciencia y Enfermería, XIV(1), 9-15.

Zahra, S. A., Neubaum, D. O., \& Naldi, L. (2007). The effects of ownership and governance on SMEs international knowledge-based resources. Small Business Economics, 29, 309-327.

Zaichkowsky, J. L. (1985). Measuring the involvement construct. Journal of Consumer Research, 12(4), 341-352.

Zhang, F., Zuo, J., \& Zillante, G. (2013). Identification and evaluation of the key social competencies for Chinese construction project managers. International Journal of Project Management, 31(5), 748-759. 